\title{
Tumour suppressor 15-hydroxyprostaglandin dehydrogenase induces differentiation in colon cancer via GLI1 inhibition
}

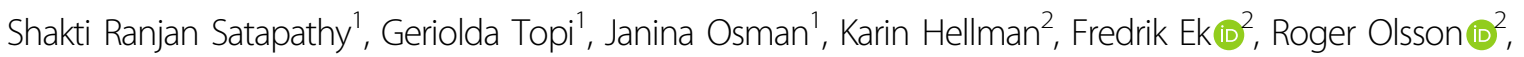 \\ Wondossen Sime ${ }^{1,3}$, Lubna M. Mehdawi ${ }^{1}$ and Anita Sjölander (1)
}

\begin{abstract}
Inflammation is an established risk factor for colorectal cancer. We and others have shown that colorectal cancer patients with elevated cysteinyl leukotriene receptor $2\left(C y s L T_{2} R\right)$ and 15-hydroxyprostaglandin dehydrogenase (15-PGDH) levels exhibit good prognoses. However, both $\mathrm{Cys}_{\mathrm{L}} \mathrm{T}_{2} \mathrm{R}$ and $15-\mathrm{PGDH}$, which act as tumour suppressors, are often suppressed in colorectal cancer. We previously reported that leukotriene $\mathrm{C}_{4}\left(\mathrm{LTC}_{4}\right)$-induced differentiation in colon cancer via CysL $T_{2} R$ signalling. Here, we investigated the involvement of Hedgehog (Hh)-GLI1 signalling, which is often hyperactivated in colorectal cancer. We found that the majority of colorectal cancer patients had high-GLI1 expression, which was negatively correlated with Cys $L_{2} R$, 15-PGDH, and Mucin-2 and overall survival compared with the low-GLI1 group. LTC $C_{4}$-induced 15-PGDH downregulated both the mRNA and protein expression of GLI1 in a protein kinase A (PKA)-dependent manner. Interestingly, the LTC $_{4}$-induced increase in differentiation markers and reduction in Wht targets remained unaltered in GLI1-knockdown cells. The restoration of GLI1 in 15-PGDH-knockdown cells did not ameliorate the $\mathrm{LTC}_{4}$-induced effects, indicating the importance of both 15-PGDH and GLI1. LTC 4 -mediated reduction in the DCLK1 and LGR5 stemness markers in colonospheres was abolished in cells lacking 15-PGDH or GLI1. Both DCLK1 and LGR5 were highly increased in tumour tissue compared with the matched controls. Reduced Mucin-2 levels were observed both in zebrafish xenografts with GL/1-knockdown cells and in the cysltr2 ${ }^{-1-}$ colitis-associated colon cancer (CAC) mouse model. Furthermore, GLI1 expression was positively correlated with stemness and negatively correlated with differentiation in CRC patients when comparing tumour and mucosal tissues. In conclusion, restoring 15-PGDH expression via CysL $T_{2} R$ activation might benefit colorectal cancer patients.
\end{abstract}

\section{Introduction}

Colorectal cancer (CRC), one of the most prevalent cancers in the world, has a high metastatic efficacy and a low 5-year survival rate ${ }^{1}$. A nontargeted therapeutic approach combined with late diagnosis leads to poor prognosis and treatment failure. More than $85 \%$ of CRC

Correspondence: Anita Sjölander (anita.sjolander@med.lu.se)

${ }^{1}$ Cell and Experimental Pathology, Department of Translational Medicine, Lund University, Skåne University Hospital, Malmö, Sweden

${ }^{2}$ Chemical Biology \& Therapeutics Group, Department of Experimental Medical Science, Lund University, Lund, Sweden

Full list of author information is available at the end of the article cases exhibit anomalous APC/Wnt/ $/$-catenin signalling, which regulates the progression of CRC by adopting different intracellular mechanisms, thus affecting cancer stem cells and interactions with the tumour microenvironment. Hedgehog $(\mathrm{Hh})$ signalling, which regulates differentiation under physiological conditions, has attracted attention because of its emerging role in the promotion and maintenance of $\mathrm{CRC}^{2-4}$. In the untransformed colon, Hh ligands are secreted by epithelial cells targeting mesenchymal cells as a classic paracrine Hh signalling pathway to ensuring the proper size and location of the crypt-villus axis ${ }^{5}$, as also observed in other tissues ${ }^{6}$.

\section{(c) The Author(s) 2020}

(c) (i) Open Access This article is licensed under a Creative Commons Attribution 4.0 International License, which permits use, sharing, adaptation, distribution and reproduction cc) in any medium or format, as long as you give appropriate credit to the original author(s) and the source, provide a link to the Creative Commons license, and indicate if changes were made. The images or other third party material in this article are included in the article's Creative Commons license, unless indicated otherwise in a credit line to the material. If material is not included in the article's Creative Commons license and your intended use is not permitted by statutory regulation or exceeds the permitted use, you will need to obtain permission directly from the copyright holder. To view a copy of this license, visit http://creativecommons.org/licenses/by/4.0/. 
In CRC, abnormal Hh signalling functions in a liganddependent manner and is activated in human CC cell lines ${ }^{7}$ and xenograft models ${ }^{4}$. However, the role of $\mathrm{Hh}$ signalling and its importance in cell survival in CRC are not well defined. Although some previous studies have failed to derive a positive correlation between Hh signalling and CRC initiation and maintenance ${ }^{8,9}$, major bodies of evidence point to a positive correlation ${ }^{3,4,7}$. Moreover, previous reports have suggested high activity of the Hh-SMO-GLI axis in CRC cell survival and metastasis, which is coordinated by either canonical signalling (via $\mathrm{SMO}$ ) or a non-canonical mode of activation (via the RAS-MAP kinase pathway $)^{4,7}$. Within these pathways, the most prominent factors are glioma-associated oncogene homologue (GLI) 1 and 2 and the transcriptional regulators downstream of SMO, which keep the oncogenic pathway active.

CRC, which is considered to be an inflammationassociated cancer, is greatly influenced by inflammatory mediators, such as leukotrienes and prostaglandins, which belong to the G-protein-coupled receptor family. The cysteinyl leukotriene receptors (CysLTRs) CysLT $_{1} R$ and $\mathrm{CysLT}_{2} \mathrm{R}$ are activated by binding with their high-affinity ligands leukotriene $\mathrm{D}_{4}$ and $\mathrm{C}_{4}\left(\mathrm{LTC}_{4}\right)$, respectively ${ }^{10,11}$. These proinflammatory lipid mediators are derived from the arachidonic acid pathway via 5-lipoxygenase, and they play crucial roles in pathological inflammation, such as that observed in inflammatory bowel disease. We previously showed that elevated $\mathrm{CysLT}_{1} \mathrm{R}$ levels were associated with poor prognoses in CRC patients, while patients with high CysLT $_{2} \mathrm{R}$ expression had better prognoses $^{12}$. Another important group of eicosanoids are prostaglandins (PGs), which are produced via the COX-2 pathway. The upregulation of COX-2 in CRC increases the $\mathrm{PGE}_{2}$ level, which promotes cancer cell proliferation, angiogenesis, survival, migration, and invasion; these are important hallmarks of cancer ${ }^{13,14}$.

The tumour suppressor 15-hydroxyprostaglandin dehydrogenase (15-PGDH) is an enzyme responsible for the degradation of $\mathrm{PGE}_{2}$ into an inactive metabolite ${ }^{15}$. 15PGDH is abundantly expressed in normal colon mucosa, but its expression is lost in CRC cells ${ }^{16,17}$, leading to disease progression. Recently, researchers have explored the efficacy of 15-PGDH as a potential antitumour agent against colon cancer ${ }^{18-21}$. In a recent study, we established that $15-\mathrm{PGDH}$ is induced by $\mathrm{LTC}_{4}$ via $\mathrm{CysLT}_{2} \mathrm{R}$ signalling by phosphorylating c-Jun $\mathrm{N}$-terminal kinase and AP-1 to induce 15-PGDH promoter activity and further guide colon cancer cells toward redifferentiation ${ }^{20}$. However, the detailed mechanism underlying this phenomenon remains unclear.

In this study, we elucidated the mechanism by which $\mathrm{LTC}_{4}$-induced 15-PGDH promotes differentiation in colon cancer cells through CysLT $_{2} \mathrm{R}$ activation with the involvement of Hh-GLI signalling. We observed that GLI1 was involved in the regulation of the redifferentiation and reduction in stemness induced by $\mathrm{LTC}_{4}$ via $15-$ PGDH in colon cancer cells.

\section{Results \\ GLI1 expression is negatively correlated with CysLT2R, 15- PGDH, and Mucin-2 expression in CRC patients}

To elucidate the regulatory activity of GLI1 on the antitumorigenic proteins CysLT $_{2} \mathrm{R}$ and 15-PGDH and the differentiation marker Mucin-2 in colon cancer, we used a tissue microarray (TMA) of primary CRCs from 326 patients $^{22}$. After IHC analysis, we found only five patients with negative GLI1 staining, 50 patients with weak staining intensity, 168 patients with moderate staining intensity, and 33 patients with strong staining intensity. The mean \pm standard deviation (SD) of the immunoreactive score (IRS) for GLI1 expression was $6.3 \pm 1.6$. Then, we grouped the patients with negative and weak staining intensity and defined them as the low-GLI1 expression group $(n=55)$, and those with moderate and strong staining intensity were defined as the high-GLI1 expression group $(n=201$; Fig. 1a). Seventy patients had missing or incomplete cores and were excluded from the final analysis.

After IHC evaluation of $\mathrm{CysLT}_{2} \mathrm{R}$ and 15-PGDH, we observed that patients with high-GLI1 expression had significantly lower levels of $\mathrm{CysLT}_{2} \mathrm{R}$ (IRS $5.4 \pm 1.7$ ) and 15-PGDH (IRS $4.5 \pm 1.3$ ) expression than those with lowGLI1 expression (Fig. 1b-d). Furthermore, there was a significant negative correlation between GLI1 and 15PGDH $(r=-0.31, P<0.0001)$, suggesting hyperactivated Hh-GLI signalling with suppressed 15-PGDH (Fig. 1e). However, no significant correlation was found between GLI1 and CysLT ${ }_{2}$ R expression (Fig. 1e).

On the other hand, patients with low-GLI1 expression had a significantly higher IRS for $\mathrm{CysLT}_{2} \mathrm{R}(6.0 \pm 1.7)$ and 15-PGDH (5.2 \pm 1.8$)$ expression (Fig. 1c, d), which indicates the adverse effects of $\mathrm{CysLT}_{2} \mathrm{R} / 15-\mathrm{PGDH}$ axis activation on GLI1. We also noticed that the majority of patients exhibited high-GLI1 expression (201/256 patients), and when stratified according to the tumournode-metastasis (TNM) staging, a significantly stronger association for the patients with TNM stages III and IV was found (Fig. 1e). In addition to the above observations, transcriptome data from a public database $\mathrm{e}^{23}$ also suggested a significant negative correlation between GLI1 and the tumour suppressors CYSLTR2 (Fig. 1f) and HPGD (15-PGDH), (Fig. 1g).

Importantly, we observed that patients with low-GLI1 expression had a $58 \%$ lower risk of overall mortality (hazard ratio $=0.42 ; 95 \% \mathrm{CI}, 0.21-0.83$ ) than patients with high-GLI1 expression after adjusting for age and TNM stage (Fig. 1h; the unadjusted survival curve is provided in Supplementary Fig. S1A). The median follow- 


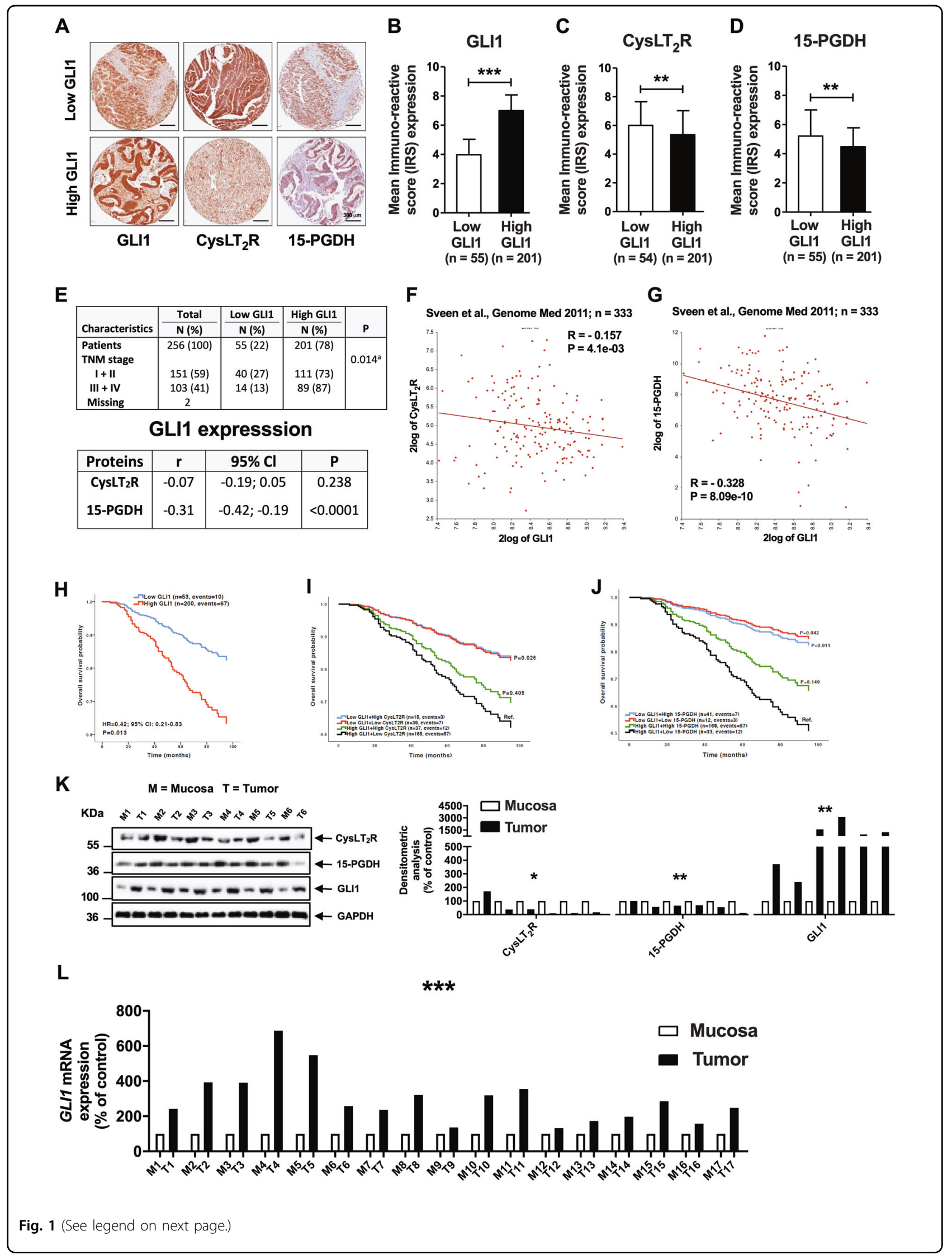


(see figure on previous page)

Fig. $1 \mathrm{GLI} 1$ expression exhibited a negative correlation with $\mathrm{CysLT}_{2} \mathrm{R}$ and 15-PGDH expression in colorectal cancer patient tissues.

a Matched pair immunohistochemistry (IHC) images of GLI1, CysLT 2 R, and 15-PGDH expression in patients with low- and high-GLI1 expression, shown at $\times 20$ magnification. The staining immunoreactivity was quantified by the mean immunoreactive score (IRS), calculated according to the following formula: IRS = (staining intensity) $\times(\%$ of stained cells). The mean IRS for the groups of patients with low- and high-GLI1 expression. $\mathbf{b}$ The Y-axis represents IRS for GLI1 in these patients. $\mathbf{c}$ CysLT 2 R and $\mathbf{d}$ 15-PGDH expression according to patients with low- and high-GLI1 expression in CRC tissue. e Distribution of tumour-node-metastasis (TNM) stages of CRC according to low- and high-GLI1 expression. Pairwise Pearson correlation coefficient ( $r$ ) between the expression of GLI1 and that of CysL $T_{2} R$ and 15-PGDH. P value according to chi-square test. XY-scatter plots showing mRNA levels of $\mathbf{f}$ GLI1 and CysLT $R$ and $\mathbf{g}$ GLI1 and 15-PGDH (HPGD) gene expression from a public database containing 333 CRC patients. Kaplan-Meier curves for overall survival adjusted for age and TNM stage for patients with $\mathbf{h}$ low- and high-GLI1 expression and subgroups of patients with both GLI1 and $\mathbf{i}$ CysLT 2 R or $\mathbf{j} 15-P G D H$ expression compared by the log-rank test. The last patient group served as the reference category. $\mathbf{k}$ Western blot analysis showing the protein expression of CysLT $R$, 15-PGDH and GLI1 in matched pairs of six patients with normal (N) and tumour ( $(T)$ areas. KDa indicated on the left side of the immunoblots (KDa 55, 36, 100,36) represents protein size markers. Graphical representation of the densitometric analysis showing the relative protein expression for Cys $\mathrm{T}_{2} \mathrm{R}, 15-\mathrm{PGDH}$, and GLI1 in matched pairs of patient samples $(n=6)$ from normal mucosa (M, white) and tumour ( $T$, black) areas. I qRT-PCR analysis of GL/1 in matched pairs of normal mucosa ( $M$, white) and tumour ( $T$, black) tissues from CRC patients $(n=17)$. Scale bar as indicated in the images. Data represent the mean \pm SD, ${ }^{*} P<0.05,{ }^{* *} P<0.01,{ }^{* * *} P<0.001$, Mann-Whitney test.

up time was 69.5 months (5.8 years), with 79 total events. This suggests a prominent role of GLI1 in colon carcinogenesis.

We have previously shown that CRC patients with low$\mathrm{CysLT}_{2} \mathrm{R}$ and/or low-15-PGDH expression have a poor prognosis $^{12,20}$. In this study, we noted that patients with high-GLI1 expression coupled with either low-CysLT ${ }_{2} R$ expression or low-15-PGDH expression had poorer prognoses than patients with either low-GLI1 and low-15PGDH expression or low-GLI1 and low-CysLT ${ }_{2} R$ expression (Fig. 1i, j). Western blot analysis of six matched pairs of CRC patients showed significantly higher protein expression of $\mathrm{CysLT}_{2} \mathrm{R}$ and 15-PGDH in normal tissue than in matched tumour tissue (Fig. 1k). However, GLI1 showed elevated expression in tumour tissue compared with matched normal tissue (Fig. 1k).

Furthermore, the mRNA analysis of these paired tumour tissues with matched mucosa tissues from CRC patients $(n=17)$ showed significantly higher mRNA expression of GLI1 in the tumour tissue compared with its matched normal mucosa (the normal mucosa as reference set to 100 and tumour tissue 298.58 \pm 35.69 (mean \pm SEM), Mann-Whitney test, $P<0.001$, Fig. 1l). Furthermore, the MUC2 (MUCIN-2) mRNA analysis of these paired tumour tissues $(\mathrm{T})$ and matched normal mucosa (M) showed significantly lower mRNA expression of $M U C 2$ in the tumour tissue compared with its matched normal mucosa (the normal mucosa as reference set to 100 and tumour tissue $92.60 \pm 34.91$ (mean \pm SEM), Mann-Whitney test, $P<0.001$, Fig. 2a). A representative matched pair of high- and low-GLI1 and the corresponding Mucin-2 is shown (Fig. 2b). We observed that patients with high-GLI1 expression had lower levels of Mucin-2 expression than those with low-GLI1 expression (Fig. 2c), and by combining these data, a significant negative correlation between elevated expression of GLI1 and decreased expression of Mucin-2 in tumour tissues was found (Fig. 2d). Moreover, grouping the patients into mucinous $(n=55)$ and non-mucinous $(n=200)$ types revealed that $75 \%$ of patients (151/200) with nonmucinous status had high-GLI1 expression, suggesting a negative correlation with Mucin-2-expressing cells, while $91 \%$ of patients $(50 / 55)$ in the mucinous category showed high-GLI1 expression (Fig. 2d). Furthermore, we found a significant negative correlation between GLI1 and the differentiation marker Mucin-2 expression in these CRC patients $(n=158$; Fig. 2 e). We found a better overall survival for patients with low-GLI1 than those with highGLI1 expression regardless of Mucin-2 expression (Fig. 2e). Taken together, these results suggest that GLI1 expression is negatively correlated with $\mathrm{CysLT}_{2} \mathrm{R}, 15$ PGDH, and Mucin-2 expression but positively with the mucinous status of the patients.

\section{Cys $L T_{2} R$ is essential for differentiation in a colitis- associated colon cancer-CAC-mouse model}

To further validate the role of $\mathrm{CysLT}_{2} \mathrm{R}$ in promoting differentiation in CRC, we adopted an inflammatory mouse model that was induced by azoxymethane (AOM) and dextran sodium sulfate (DSS). Briefly, C57BL/6N wild-type and cysltr $2^{-1-}$ mice were subjected to AOM and two 2\% DSS cycles $^{24}$ (Fig. 2f) as described in the Materials and Methods section. We found that all mice formed polyps in the colon regardless the phenotype but cysltr $2^{-l-}$ mice $(n=13)$ developed significant larger polyps ( $\geq 1.5 \mathrm{~mm}, P=0.0159)$ in the colon compared with their wild-type ( $w t ; n=11)$ littermates (Supplementary Fig. S1B, C). This result indicates that cysltr $2^{-1-}$ mice develop a more progressive disease. Supplementary Fig. S1 shows a representative image of colon polyps (Fig. S1D) and a likewise representative Haemotoxylin and Eosin-stained image (Fig. S1E) showing premalignant areas, aberrant crypt foci and metaplasia areas from both $w t$ and cysltr2 $2^{-/-}$mice colon.

Colon tissue sections from $w t$ mice showed abundant Mucin-2-expressing cells compared with sections from 


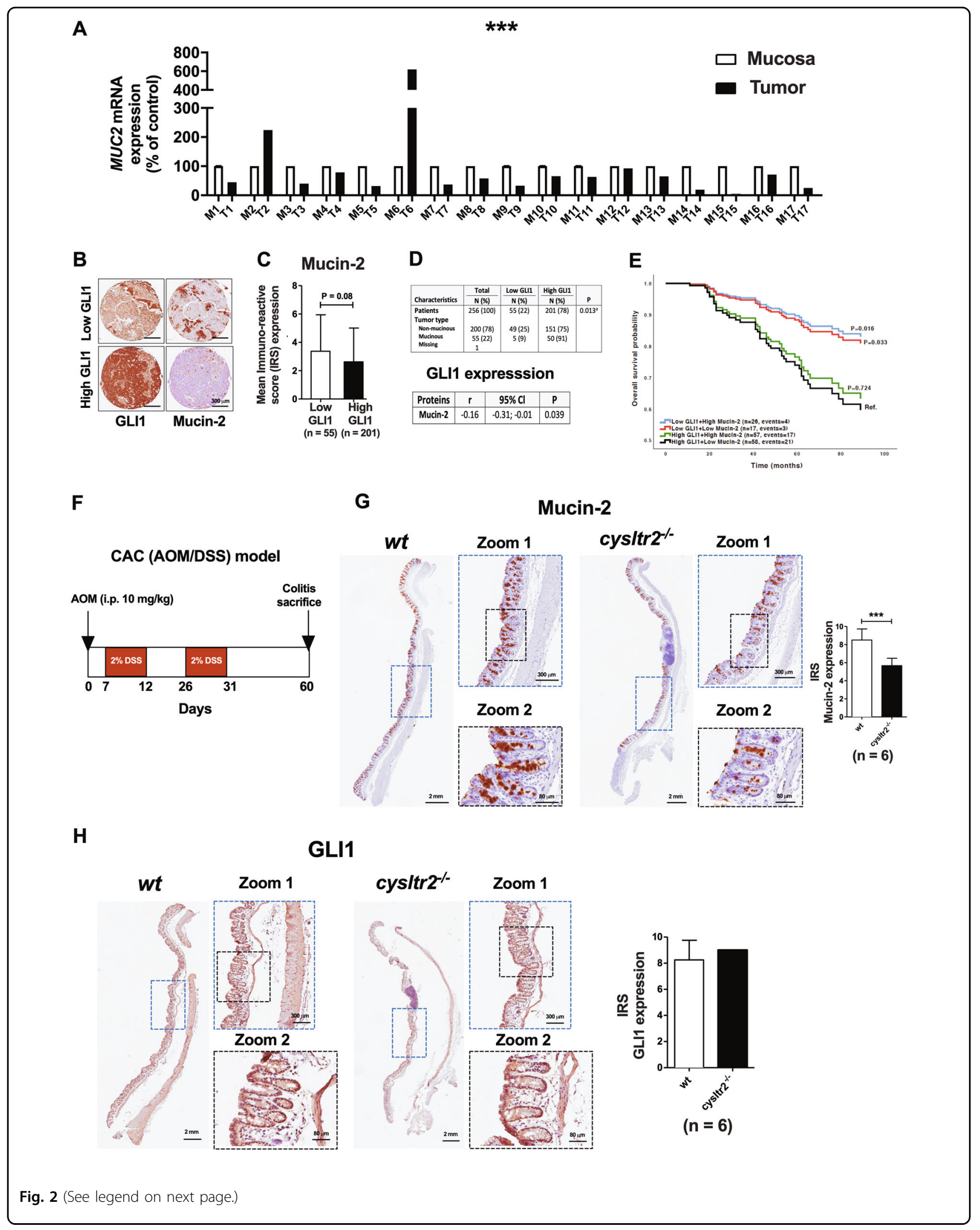


(see figure on previous page)

Fig. 2 GLI1 expression was negatively correlated with differentiation. a qRT-PCR analysis of MUCIN-2 in matched pairs of normal mucosa (M, white) and tumour (T, black) tissues from CRC patients $(n=17)$. b Immunohistochemistry (IHC) images for GLI1 and Mucin-2 expression in matched paired tissue samples from colorectal cancer (CRC) patients with low- and high-GLI1 expression represented at $\times 20$ magnification. c Quantification of the staining immunoreactivity by the mean IRS for Mucin-2 expression according to patients with low- and high-GLI1 expression in CRC tissue. d Distribution of tumour type, mucinous adenocarcinomas versus non-mucinous adenocarcinomas, according to low- and high-GLI1 expression. Pairwise Pearson correlation coefficient ( $r$ ) between the expression of GLI1 and Mucin-2. P value according to chi-square test. e Kaplan-Meier curves for overall survival adjusted for age and TNM stage subgroups of patients with both GLI1 and Mucin-2 expression compared by the log-rank test. The high-GLI1 and low-Mucin-2 patient group was set as the reference category. $\mathbf{f}$ Experimental schematic of the AOM-DSS mouse model. GLI1 expression exhibited a negative correlation with differentiation in the cysltr2 ${ }^{-1-}$ AOM-DSS mouse model. Immunohistochemical evaluation showing the protein expression of $\mathbf{g}$ Mucin-2 and $\mathbf{h}$ GLI1 in wt and cys/tr2 ${ }^{-/-}$AOM-DSS-challenged mice. Graph bars showing the IRS scores for Mucin-2 and GLI1, respectively, compared between wt and cysltr $2^{-1-}, n=6$ mice/group. Scale bar as indicated in the images. Data represent the mean \pm SD, ***P $<0.001$, Mann-Whitney test.

cysltr $2^{-1-}$ mice (5.7 $\pm 0.3, n=6$; Fig. $\left.2 g\right)$. GLI1 exhibited a slightly higher but non-significant overall expression in cysltr $2^{-1-}$ mouse tissue sections compared with $w t$ tissue sections (7.5 $\pm 0.6, n=6$; Fig. $2 \mathrm{~h})$. Taken together, the above observations encouraged us to further investigate Hh-GLI signalling using both in vitro and in vivo colon cancer model systems and to delineate the involvement of $\mathrm{CysLT}_{2} \mathrm{R}$ and 15-PGDH in promoting differentiation.

\section{LTC4-induced 15-PGDH downregulates GLI1 in colon cancer cells}

We determined whether Hh-GLI signalling was involved in the $\mathrm{LTC}_{4}$-induced 15-PGDH-promoted differentiation of CC cells ${ }^{20}$. Interestingly, $\mathrm{LTC}_{4}$ stimulation significantly downregulated GLI1 expression at both the mRNA and protein levels compared with unstimulated HT-29 and Caco-2 cells (Fig. 3a, b). In addition, immunofluorescence analysis of GLI1 expression revealed a decrease in nuclear GLI1 upon $\mathrm{LTC}_{4}$ stimulation (Fig. 3c). To determine the mechanism of 15-PGDH-mediated depletion of GLI1, we examined the expression of protein kinase A (PKA), which is a known GLI1 antagonist ${ }^{25,26}$. The activation of the PKA $(\alpha / \beta / \delta)$ catalytic subunit in Caco-2 cells after stimulation by $\mathrm{LTC}_{4}$ was significantly increased, as indicated by the levels of phosphorylated PKA threonine 197, but remained unchanged in stimulated HT-29 cells (Fig. 3b). Moreover, phosphorylation at serine 338 on the catalytic subunit of PKA $(\beta)$ was increased after $\mathrm{LTC}_{4}$ stimulation in HT-29 cells but was not present in Caco-2 whole-cell lysates (Fig. 3b).

15-PGDH-specific shRNA (shHPGD) was employed to investigate the involvement of $15-\mathrm{PGDH}$ in the $\mathrm{LTC}_{4}$ mediated downregulation of GLI1 expression. Compared with cells transfected with control shRNA (shCTRL), CC cells transfected with shHPGD showed no response to $\mathrm{LTC}_{4}$ stimulation. We observed that shRNA-mediated knockdown of $H P G D$ did not affect the expression of the intestinal differentiation markers $C D X 2$ or $C D H R 2$ or the Wnt target AXIN2 (Supplementary Fig. S2A-D for HT-29 cells) and unaltered GLI1 expression at both the mRNA and protein levels (Fig. 3d-f; see Supplementary Fig. S3A-F for Caco-2 cells). Similarly, 15-PGDH knockdown reduced the effect of $\mathrm{LTC}_{4}$ stimulation on PKA activation in both HT-29 and Caco-2 cells (Fig. 3e; Supplementary Fig. S3F).

To further confirm the role of PKA as an intermediate molecule in $\mathrm{LTC}_{4}$-induced 15-PGDH-mediated downregulation of GLI1, we used H89, a PKA-specific inhibitor $(135 \mathrm{nM})$, prior to stimulating the cells with $\mathrm{LTC}_{4}$. We found that neither HT-29 nor Caco-2 cells treated with the PKA inhibitor affected $\mathrm{LTC}_{4}$-induced 15-PGDH expression at either the mRNA or protein levels. However, no significant alteration was observed in either the mRNA or protein level of GLI1 post-stimulation with $\mathrm{LTC}_{4}$ (Fig. 3g, h; Supplementary Fig. S4A, B), which was also supported by immunofluorescence analysis (Fig. 3i; Supplementary Fig. S4C). These data indicate that PKA plays a role in the 15-PGDH-mediated downregulation of GLI1 in colon cancer cells.

\section{GLI1 regulates 15-PGDH-promoted differentiation in colon cancer cells}

Next, we determined the mechanism by which $\mathrm{LTC}_{4-}$ induced 15-PGDH promoted differentiation in colon cancer cells ${ }^{20}$. Based on the above evidence, we investigated the effects of GLI1 knockdown (Fig. 4a-j for HT-29 cells and Supplementary Fig. S5A-K for Caco-2 cells) on HPGD (15-PGDH) expression. We used shGLI1 to determine possible changes in the expression of 15PGDH. However, GLI1 knockdown in these cells did not affect $\mathrm{LTC}_{4}$-induced 15-PGDH expression at either the mRNA or protein level compared with the corresponding shCTRL-transfected cells. These data indicate that the effect of $\mathrm{LTC}_{4}$ signalling on $15-\mathrm{PGDH}$ expression is independent of GLI1, suggesting that GLI1 is downstream of 15-PGDH. We next validated the involvement of GLI1 in differentiation by testing the mRNA expression of $S I$ (sucrase-isomaltase) and MUC2 (Mucin-2), which are representative intestinal differentiation markers, following $\mathrm{LTC}_{4}$ stimulation. The observed increases in the mRNA 

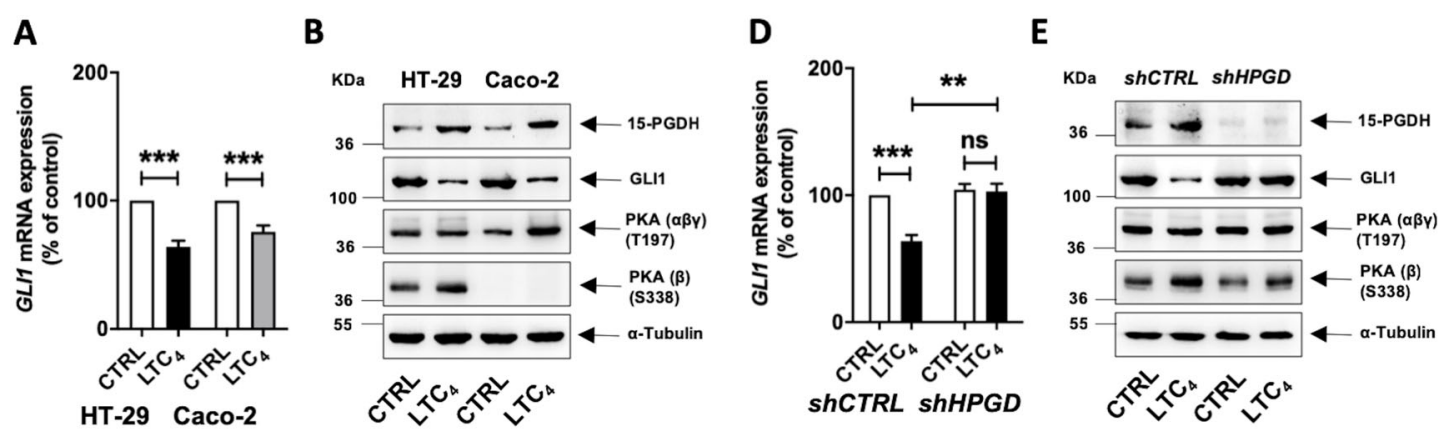

C
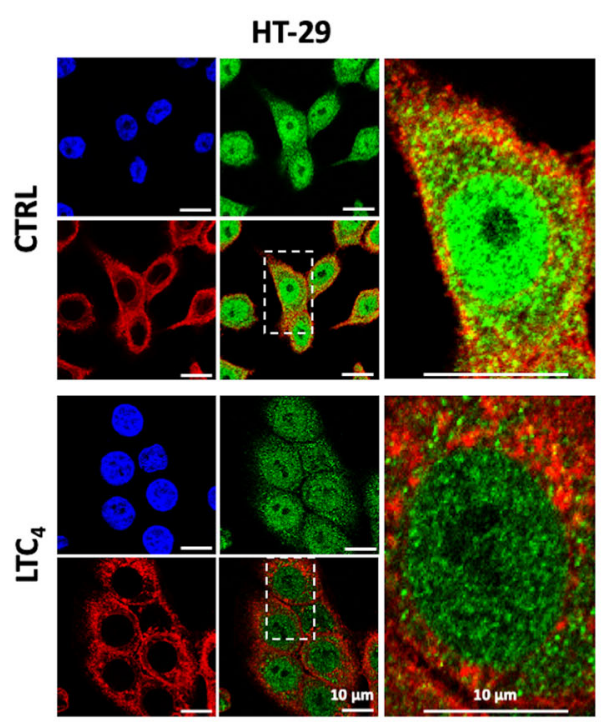

DAPI GLI1 F-actin Merged

G

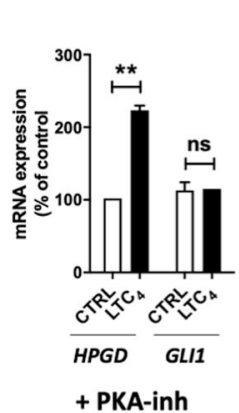

H

KDa

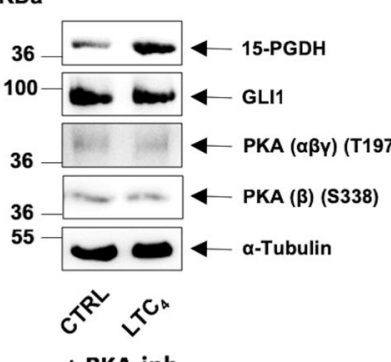

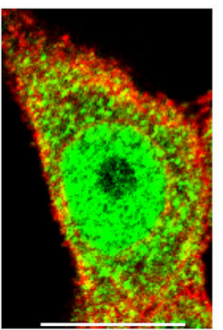

+ PKA-inh

I
$\mathbf{F}$

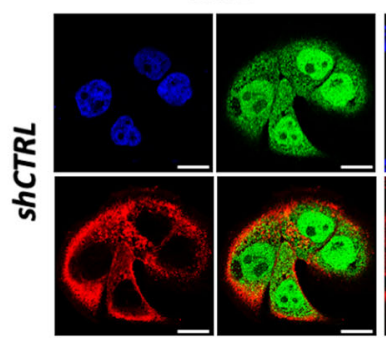

CTRL

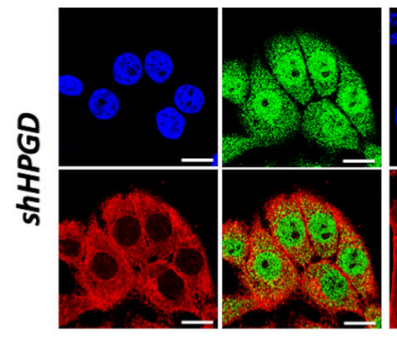

DAPI GLI1 F-actin Merged

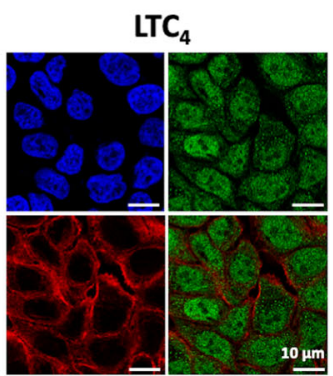

DAPI GLI1 F-actin Merged

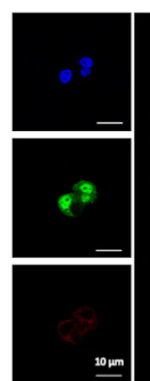

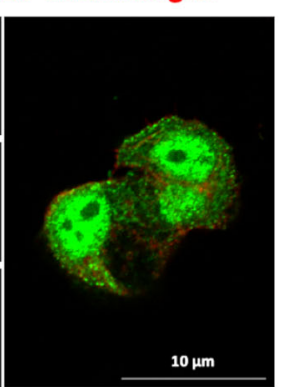

CTRL

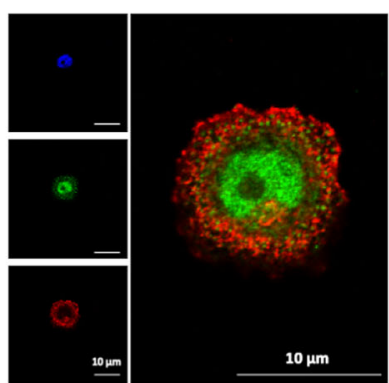

$\mathrm{LTC}_{4}$

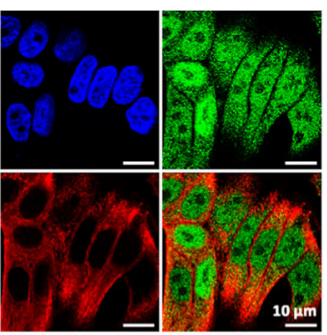

+ PKA-inh

Fig. 3 15-PGDH regulates the $\mathrm{LTC}_{4}$-mediated downregulation of $\mathrm{Hh}$-GLI signalling in colon cancer cells. a qRT-PCR analysis of GL/1 mRNA expression in HT-29 and Caco-2 cells with or without $\mathrm{LTC}_{4}$ stimulation for $48 \mathrm{~h}$. $\mathbf{b}$ Western blot analysis of 15-PGDH, GLI1, and phospho-PKA (aßY subunit and $\beta$ subunit) levels in HT-29 and Caco-2 cells with or without $L_{T C}$ stimulation. a-Tubulin served as the loading control. c Immunofluorescence analysis of GLI1 in HT-29 cells with or without LTC 4 stimulation for $48 \mathrm{~h}$. $\mathbf{d}$ qRT-PCR analysis of HT-29 cells transfected with control shRNA (shCTRL) or PGDH-specific shRNA (shHPGD) with or without LTC 4 stimulation for $48 \mathrm{~h}$. e Western blot analysis of HT-29 cells transfected with control shRNA (shCTRL) or PGDH-specific

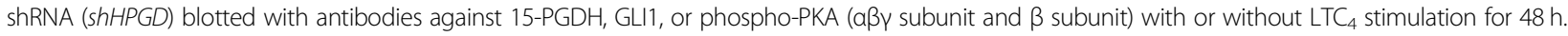
a-Tubulin served as the loading control. $\mathbf{f}$ Immunofluorescence analysis of GLI1 in HT-29 cells transfected with control shRNA (shCTRL) or PGDH-specific shRNA (shHPGD) with or without LTC 4 stimulation for $48 \mathrm{~h} . \mathbf{g}$ qRT-PCR analysis of 15-PGDH and GL17 in HT-29 cells treated with the PKA inhibitor H89 (PKAinh) for $6 \mathrm{~h}$ followed by $\mathrm{LTC}_{4}$ for $48 \mathrm{~h}$. $\mathbf{h}$ Western blot analysis showing the expression of 15-PGDH, GLI1, and phospho-PKA (aßY subunit and $\beta$ subunit) in HT-29 cells treated with the PKA inhibitor H89 (PKA-inh) for $6 \mathrm{~h}$ followed by LTC for $48 \mathrm{~h}$. i Immunofluorescence analysis of GLI1 in HT-29 cells treated with the PKA inhibitor H89 (PKA-inh) for $6 \mathrm{~h}$ followed by LTC for $48 \mathrm{~h}$. HPRT1 was used as the housekeeping gene for normalisation of the qRT-PCR gene expression data. Graphs represent the mean \pm SEM of data from 3 to 4 independent experiments, ${ }^{* *} P<0.01,{ }^{* * *} P<0.001$. 


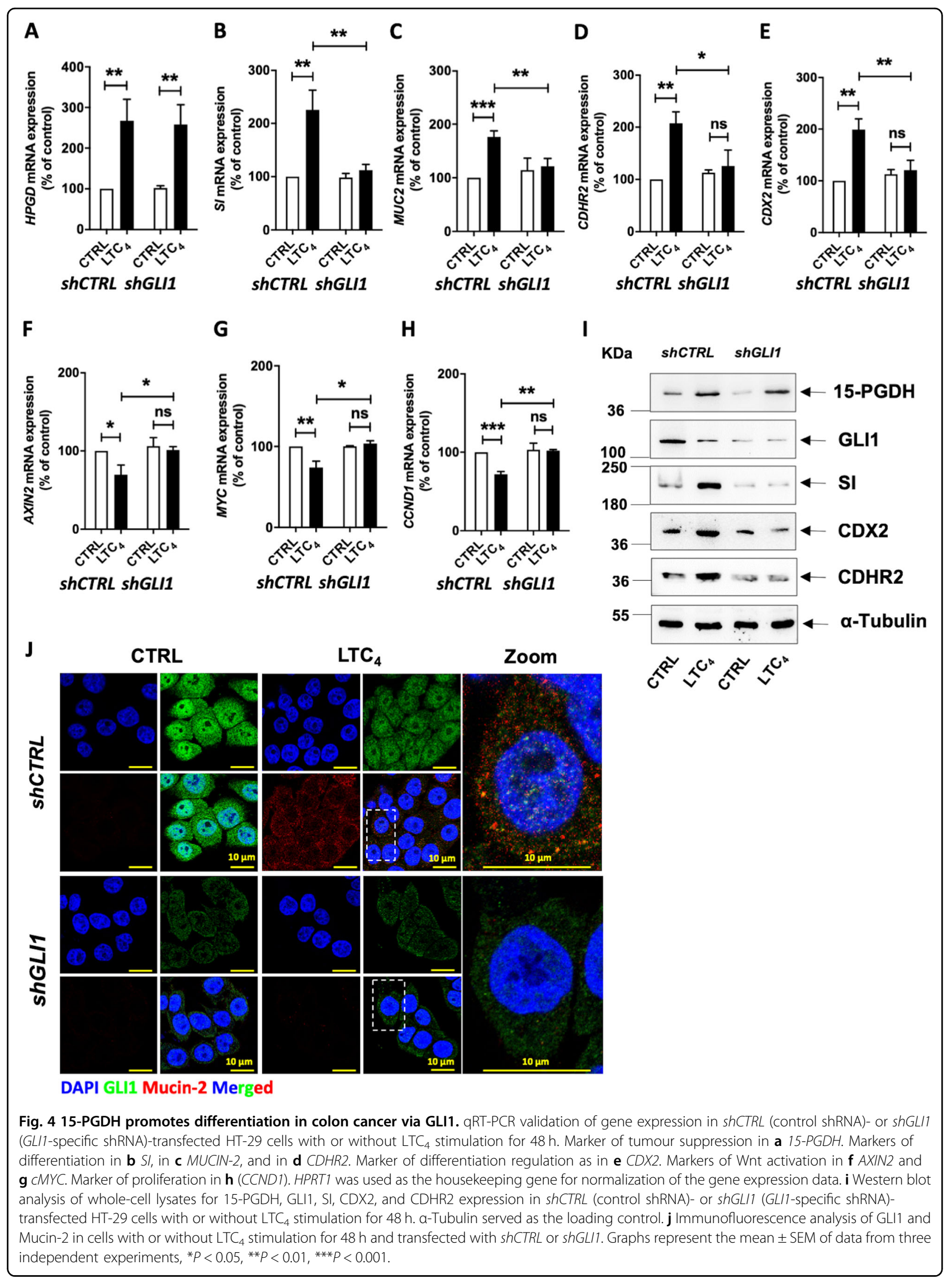


and protein expression of SI after $\mathrm{LTC}_{4}$ stimulation in both HT-29 (Fig. 4b) and Caco-2 cells (Supplementary Fig. S5C) were abolished in GLI1-knockdown cells (Fig. 4b; Supplementary Fig. S5C, J). Furthermore, similar results were observed for the mRNA expression of other differentiation markers, such as MUC2 (Fig. 4c; Supplementary Fig. S5D), and for both the mRNA and protein expression of CDHR2 and CDX2 (Fig. 4d, e; Supplementary Fig. S5E, F, J). $\mathrm{LTC}_{4}$ induced a reduction in AXIN2, a potential Wnt signalling target (Fig. 4f; Supplementary Fig. S5G). Furthermore, $M Y C$ and CCND1 mRNA expression was also abolished in GLI1-knockdown cells exposed to $\mathrm{LTC}_{4}$ (Fig. 4g, h; Supplementary Fig. S5H, I). To further validate the above observations, we performed western blot (Fig. 4i) and immunofluorescence microscopy using double staining for GLI1 and Mucin-2 in shCTRL- and shGLI1-transfected cells and found that Mucin-2 expression was downregulated in cells lacking GLI1 (Fig. 4j; Supplementary Fig. S5K). These findings support our hypothesis of a possible regulatory effect of GLI1 in LTC $_{4}$-induced, 15-PGDH-promoted differentiation in colon cancer cells.

\section{GLI1 suppresses differentiation in the absence of 15-PGDH}

We next investigated the ability of GLI1 to regulate 15PGDH by overexpressing GLI1 using the pEGFP- $h$ GLI1 construct in combination with the simultaneous knockdown of HPGD with shHPGD (Supplementary Figs. S6A, $\mathrm{B} ; \mathrm{S7A}, \mathrm{B})$. We found significant downregulation in the $\mathrm{LTC}_{4}$-induced increase in $S I, M U C 2, C D H R 2$, and $C D X 2$ mRNA expression levels in HT-29 cells (Fig. 5a-d) and also in Caco-2 cells (Supplementary Fig. S7C-F) that overexpressed GLI1 and lacked 15-PGDH compared with cells expressing the control vector $(\operatorname{shCTRL})$. We also found that the $\mathrm{LTC}_{4}$-induced reduction in AXIN2, MYC, and CCND1 mRNA was abolished, although we observed increased basal levels of these mRNAs (Fig. 5e-g; Supplementary Fig. S7G-I). We next investigated the protein expression of SI by western blot (Fig. 5h; Supplementary Fig. S7J) and Mucin-2 by immunofluorescence microscopy (Fig. 5i; Supplementary Fig. S7K). The LTC $_{4}$ induced increase in SI was abolished in HPGD-knockdown and GLI1-overexpressing cells (Fig. 5h; Supplementary Fig. S7J). A similar pattern was found regarding Mucin-2 protein expression in these cells, which was observed using immunofluorescence microscopy (Fig. 5i; Supplementary Fig. S7K). Finally, the $\mathrm{LTC}_{4}$-induced reduction in GLI1 was abolished (Fig. 5i).

\section{$\mathrm{LTC}_{4}$-induced $\mathrm{CysLT}_{2} \mathrm{R}$ signalling downregulates $\mathrm{GLI} 1$ in a 15-PGDH-dependent manner}

To determine whether $\mathrm{LTC}_{4}$ acts via $\mathrm{CysLT}_{2} \mathrm{R}$, as $\mathrm{LTC}_{4}$ is the high-affinity ligand of $\mathrm{CysLT}_{2} \mathrm{R}$, we investigated the specific involvement of CysLT ${ }_{2} \mathrm{R}$ signalling in the $\mathrm{LTC}_{4}{ }^{-}$ mediated downregulation of GLI1 in colon cancer cells. We first constructed HCT-116 cells with doxycycline (Dox)-inducible stable knockdown of $\mathrm{CysLT}_{2} \mathrm{R}$ $(\operatorname{shCYSLTR} 2)^{27}$. We examined the mRNA and protein expression levels of $\mathrm{CysLT}_{2} \mathrm{R}, 15-\mathrm{PGDH}$, and GLI1 with and without $\mathrm{LTC}_{4}$ stimulation in this cell line with Dox induction and compared them with the levels in cells grown in the absence of Dox. The mRNA expression of CYSLTR2 and HPGD was significantly upregulated (Fig. 6a, b), while GLI1 mRNA expression was downregulated in cells cultured without Dox (Fig. 6c). However, in the Dox-induced cells, GLI1 gene expression remained unaltered, most likely due to the low mRNA expression levels of CYSLTR2 and HPGD. These observations were also reflected in the levels of protein expression (Fig. 6d; Supplementary Fig. S8A-C).

To further study the involvement of $\mathrm{CysLT}_{2} \mathrm{R}$, we treated HT-29 and Caco-2 cells with the CysLT ${ }_{2} \mathrm{R}$-specific antagonist AP100984 $(1 \mu \mathrm{M})$ followed by $\mathrm{LTC}_{4}$ stimulation for $48 \mathrm{~h}^{20}$. AP100984 treatment efficiently blocked both the mRNA (Supplementary Fig. S8D, E) and protein expression (Supplementary Fig. S8F) of CysLT $\mathrm{C}_{2} \mathrm{R}$ as well as of its downstream signal 15-PGDH in HT-29 cells as well as in Caco-2 cells (Supplementary Fig. S8G-J). AP100984 pretreatment abolished the effect of $\mathrm{LTC}_{4}$ stimulation on GLI1 at both the mRNA and protein levels (Fig. 6e, f; Supplementary Fig. S8I, J). Taken together, the above results showed that $\mathrm{CysLT}_{2} \mathrm{R}$ signalling plays a role in the $\mathrm{LTC}_{4}$ induced 15-PGDH-mediated downregulation of GLI1.

\section{$\mathrm{LTC}_{4}$-induced 15-PGDH expression reduces stemness in colonospheres}

We extended our study to determine whether $\mathrm{LTC}_{4-}$ induced 15-PGDH affected stemness in colon cancer cells as well as the possible role of GLI1. We created a 3D model of multicellular colonospheres derived from colon cancer cells (Fig. 6g). We observed that shCTRL-transfected HT-29 or Caco-2 cell-derived colonospheres showed reduced numbers and sizes with $\mathrm{LTC}_{4}$ stimulation (Fig. 6). Unlike the shCTRL group, shHPGD-transfected cell-derived colonospheres showed increases in number and size. However, the absence of GLI1 in shGLI1-transfected cell-derived colonospheres resulted in no significant alteration in size or number even after $\mathrm{LTC}_{4}$ stimulation. The mRNA expression levels of the colon cancer-specific stemness markers DCLK1, LGR5, and ALDH1A1 were elevated in HT-29 as well as in Caco2 cell-derived colonospheres and were downregulated after LTC $_{4}$ stimulation (Fig. 6i; Supplementary Fig. $\mathrm{S} 9 \mathrm{~A}-\mathrm{H})$. The mRNA and protein levels of the cancer stem cell markers DCLK1 and ALDH1A1 remained unchanged in both shHPGD- and shGLI1-knockdown HT-29 and Caco-2 cell-derived colonospheres, even after $\mathrm{LTC}_{4}$ stimulation, compared with their unstimulated counterparts 


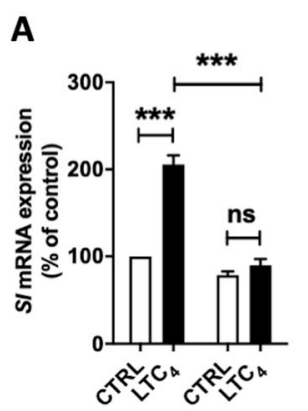

ShCTRL ShHPGD $+h G L I 1$

F

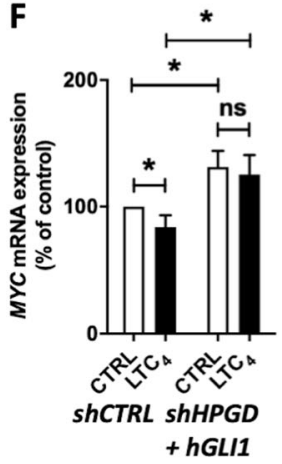

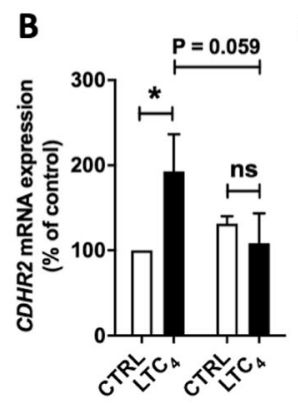

ShCTRL ShHPGD

$+h G L 1$

G

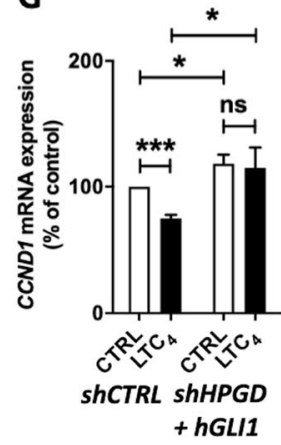

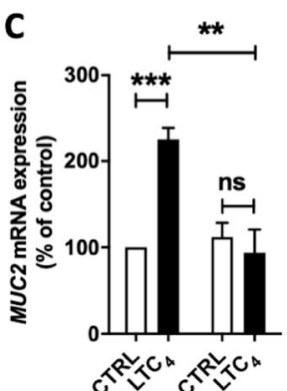

ShCTRL ShHPGD

$+h G L I 1$

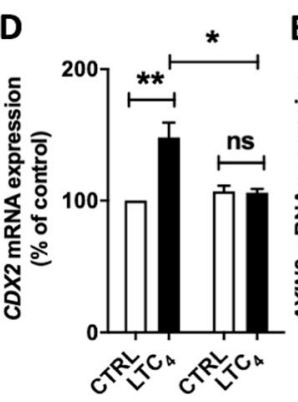

ShCTRL ShHPGD

$+h G L / 1$

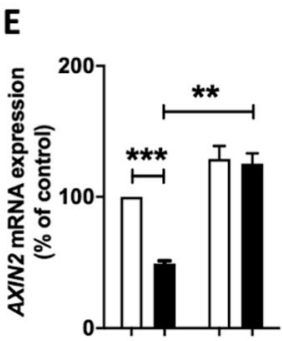

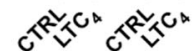

ShCTRL ShHPGD

$+h G L 1$

H

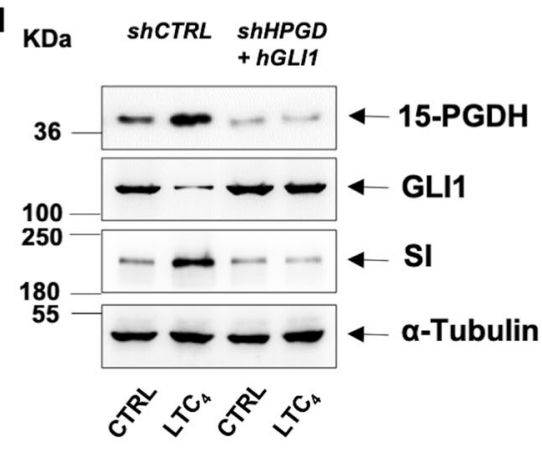

I

CTRL

LTC $_{4}$
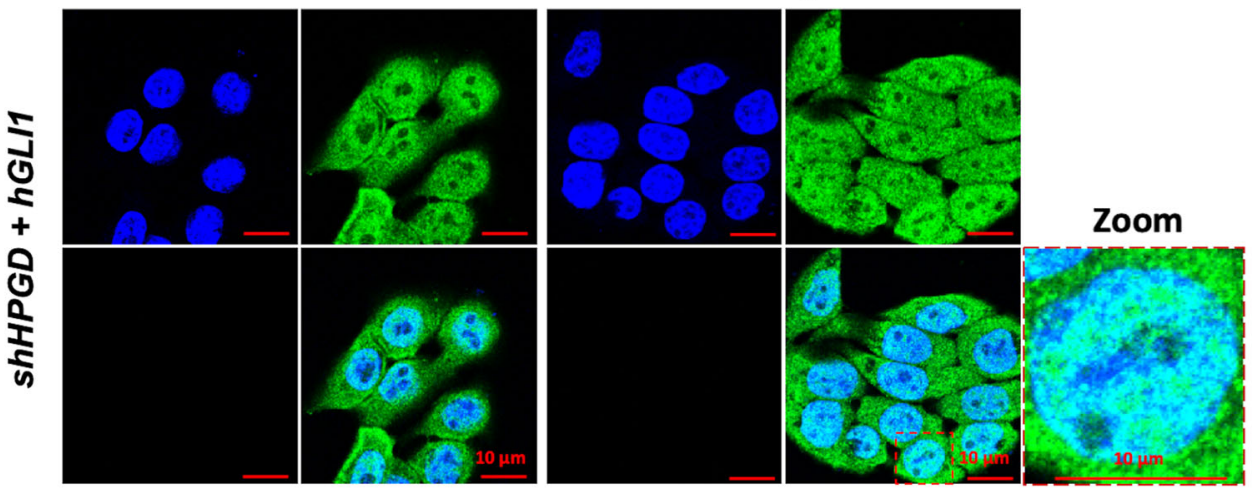

DAPI GLI1 Mucin-2 Merged

Fig. 5 GLI1 negatively regulates the differentiation and promotes the proliferation of colon cancer cells in the absence of 15-PGDH. HT-29 cells were either transfected with shCTRL alone or co-transfected with shHPGD and pEGFP-hGL/1 followed by LTC 4 stimulation for $48 \mathrm{~h}$. qRT-PCR analysis of the differentiation markers a SI, b MUCIN-2 and c CDHR2, $\mathbf{d}$ the differentiation regulation marker CDX2, e the Wnt activation marker AXIN2, $\mathbf{f}$ the pro-oncogene $C M Y C$ and $\mathbf{g}$ the proliferation marker CCND1. $\mathbf{h}$ Western blot analysis of whole-cell lysates for 15-PGDH, GLI1, and SI expression.

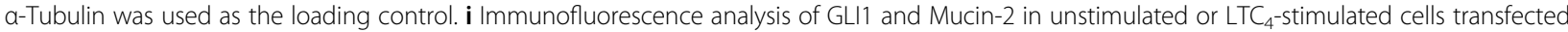
with shHPGD or shGLI1. HPRT1 was used as the housekeeping gene for normalization of the GRT-PCR gene expression data. Graphs represent data from 3 to 4 independent experiments and represent the mean \pm SEM, ${ }^{*} P<0.05,{ }^{* *} P<0.01,{ }^{* * *} P<0.001$.

(Fig. 6i-k; Supplementary Fig. S9A-H). GLI1 gene and protein expression in HT-29- and Caco-2-derived colonospheres was also downregulated after $\mathrm{LTC}_{4}$ stimulation. We next analysed the mRNA expression levels in tumour and adjacent mucosa samples from 17 paired CRC patients, which also suggested a significantly positive correlation between GLI1 and the stemness markers
DCLK1 and LGR5 with elevated expression of all three genes in tumour tissues (Figs. 1l, 6l-n). The normal mucosa as reference, set to 100 and the tumour tissue for DCLK1 $563.11 \pm 166.84$ and LGR5 $722.40 \pm 215.94$ (mean \pm SEM) respectively, Mann-Whitney test, $P<0.001$. As expected, the expression level of $D C L K 1$ showed a negative correlation with CYSLTR2 and HPGD expression 


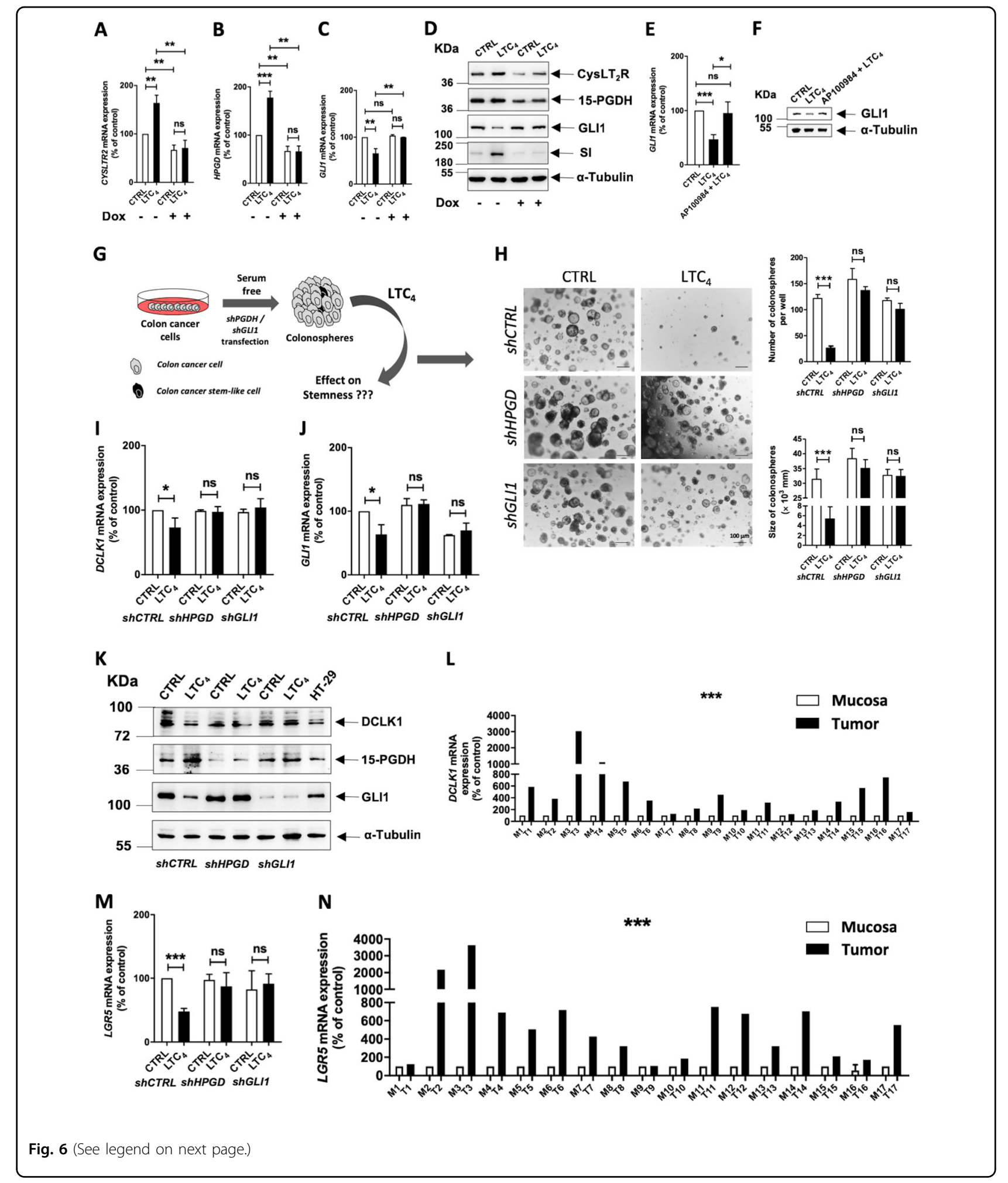

from our previously published results (see ref. ${ }^{20}$ ), while MUC2 had a positive correlation (Fig. $2 \mathrm{c})^{20}$. This suggests that a poorly differentiated tumour could occur due to enriched stemness.
LTC $_{4}$-induced 15-PGDH promotes differentiation in zebrafish xenografts

Next, we used the zebrafish xenograft model ${ }^{28}$ to further evaluate and visualise the differentiation-promoting 
(see figure on previous page)

Fig. 6 LTC $_{4}$-induced 15-PGDH expression negatively regulates GLI1 via CysLT ${ }_{2} \mathbf{R}$. Graphs showing qRT-PCR analysis of a CYSLTR2, b 15-PGDH, and c GLI1 mRNA expression in HCT-116 cells with stable transfection of doxycycline-regulated shCYSLTR2 cultured with or without doxycycline $(1 \mu \mathrm{M})$ treatment followed by $\mathrm{LTC}_{4}(40 \mathrm{nM})$ stimulation for $48 \mathrm{~h}$. d Western blot analysis of CysLT 2 R, 15-PGDH, GLI1, and SI expression in whole-cell lysates. a-Tubulin served as the loading control. e qRT-PCR analysis showing mRNA expression of GLI1 and $\mathbf{f}$ western blot analysis of GLI1 protein expression in HT-29 cells stimulated with or without $\mathrm{LTC}_{4}$ and with or without AP100984 (a CysLT $R$ antagonist). HPRT1 was used as the housekeeping gene, and a-tubulin was used as the loading control in the western blot assay. $\mathbf{g}$ Schematic illustration of colonosphere formation. GLI1 regulates the effect of $\mathrm{LTC}_{4}$ on stemness in multicellular colonospheres. The cells were cultured in ultra-low-attachment conditions on matrigel containing serumfree medium for 14 days. $\mathbf{h}$ Representative images of colonospheres from HT-29 cells transfected with shCTRL, shHPGD, or shGL/1 and stimulated or not stimulated with $\mathrm{LTC}_{4}$. Bar graphs showing the number of colonospheres formed per well and the size of colonospheres with or without $\mathrm{LTC}_{4}$ stimulation and comparing the shCTRL-, shHPGD-, and shGL/1-transfected groups. QRT-PCR analysis of the stemness markers $\mathbf{i} D C L K 1, \mathbf{j} G L / 1$, and m LGR5 in colonospheres derived from shCTRL-, shHPGD-, or shGL/1-transfected HT-29 cells with or without LTC stimulation for $48 \mathrm{~h}$. $\mathbf{k}$ Western blot analysis showing the expression of DCLK1, 15-PGDH, and GLI1 in transfected HT-29 cells as indicated. a-Tubulin served as the loading control. qRTPCR analysis of I DCLK1 and $\mathbf{n} L G R 5$ in matched pairs of mucosa (M) and tumour (T) tissues from CRC patients $(n=17)$. HPRT1 was used as the housekeeping gene for normalization of the qRT-PCR gene expression data. Data represent the mean \pm SEM from 4 to 5 independent experiments, ${ }^{*} P<0.05,{ }^{* *} P<0.01,{ }^{* * *} P<0.001$.

role of 15-PGDH in colon cancer. Transgenic zebrafish $\mathrm{Tg}$ (fli1:EGFP) embryos were injected with unstimulated or $\mathrm{LTC}_{4}$-stimulated HT-29 cells and incubated for $48 \mathrm{~h}$ (Fig. 7a). We found a twofold increase in Mucin-2 expression in zebrafish xenografts injected with $\mathrm{LTC}_{4}$-stimulated HT-29 cells compared with zebrafish xenografts injected with unstimulated cells (Fig. 7b). Furthermore, Mucin-2 expression was downregulated in zebrafish embryos injected with shGLI1-transfected HT-29 cells even after $\mathrm{LTC}_{4}$ stimulation (Fig. 7c). This observation further strengthened the conclusion that GLI1 is involved in regulating the redifferentiation of CC cells.

\section{Discussion}

Previously, we reported an antitumour effect of $\mathrm{LTC}_{4}$ via the induction of 15-PGDH. In this study, we focused on the role of GLI1 in the context of $\mathrm{LTC}_{4} / \mathrm{CysLT}_{2} \mathrm{R}$ signalling in colon cancer. In addition to hyperactivated $\mathrm{Wnt} / \beta$-catenin signalling, canonical Hh signalling acts as a prominent regulator of colonic tumorigenesis ${ }^{4,7}$. Canonical Hh signalling involves the coordinated action of the patch family transmembrane receptor $\mathrm{PTCH}$, the intermediate molecule Smoothened (SMO) and GLI, which is a transcriptional regulator downstream of sonic hedgehog (SHH). Under normal physiological conditions, Hh signalling regulates embryogenesis, development, and differentiation $^{29}$; lon carcinoma ${ }^{4,7}$. Although GLI1 has been targeted in other solid tumours ${ }^{30-32}$, it has never been addressed whether Hh-GLI inhibition could promote differentiation.

In this study, the promotion of differentiation in colon cancer cells was found to occur via the inhibition of canonical Hh/GLI1 signalling. We demonstrated that the induction of $15-\mathrm{PGDH}$ by $\mathrm{LTC}_{4} / \mathrm{CysLT}_{2} \mathrm{R}$ signalling in cells downregulated GLI1, a prominent downstream target of $\mathrm{Hh}$ signalling. $\mathrm{LTC}_{4}$ induced significant phosphorylation of the subunits $\beta$ (S338) and/or $\alpha \beta \gamma$ (T197) of
PKA, a well-known GLI1 antagonist, in HT-29 and Caco2 cells. The involvement of PKA was further confirmed by using a specific inhibitor (H89). Although these cells exclusively engage different subunits of PKA, these findings provide substantial evidence that PKA is an intermediate player involved in the 15-PGDH-mediated decrease in GLI1 expression. The decrease in GLI1 expression led to the redifferentiation and reduction in the proliferation marker CYCLIND1 in cells. Furthermore, in multicellular colonospheres, $\mathrm{LTC}_{4}-\mathrm{CysLT}_{2} \mathrm{R}$ signalling decreased the expression of the stemness markers ALDH1A1, DCLK1, and LGR5 in a 15-PGDH/GLI1dependent manner, which supported the conclusions of a previous study ${ }^{4}$ in which Varnat et al. showed the exclusive involvement of Hh-GLI1 signalling in the enrichment of CC stemness. Moreover, we used an AOM/DSSinduced CAC model and validated the involvement of $\mathrm{CysLT}_{2} \mathrm{R}$ signalling in coordinating this process. In cells obtained from mouse intestine and colon cancer patients, we identified GLI1 as a downstream regulator of 15PGDH-induced redifferentiation. Transgenic zebrafish xenografted with HT-29 cells revealed a GLI1-dependent increase in Mucin-2-expressing cells after $\mathrm{LTC}_{4}$ stimulation. When we compared the expression levels of GLI1 and DCLK1 or LGR5 between matched pairs of normal and cancer tissues from CRC patients, we found that their expression levels were negatively correlated with $M U C I N-$ 2 expression in cancer tissue.

The $\mathrm{PGE}_{2}$-degrading enzyme 15-PGDH has been recently studied to determine its antitumour potential in solid tumours such as pancreas and lung cancer ${ }^{33,34}$ in addition to colon cancer ${ }^{19,20,35}$. Some studies have focused on the antitumour role of 15-PGDH through $\mathrm{PGE}_{2}$ degradation ${ }^{36,37}$, whereas the findings of other studies have implied a differentiation-promoting role ${ }^{20}$. Two consecutive studies by Kangwan et al. showed the antitumour role of $\mathrm{SHH}$ inhibitors against colonic 


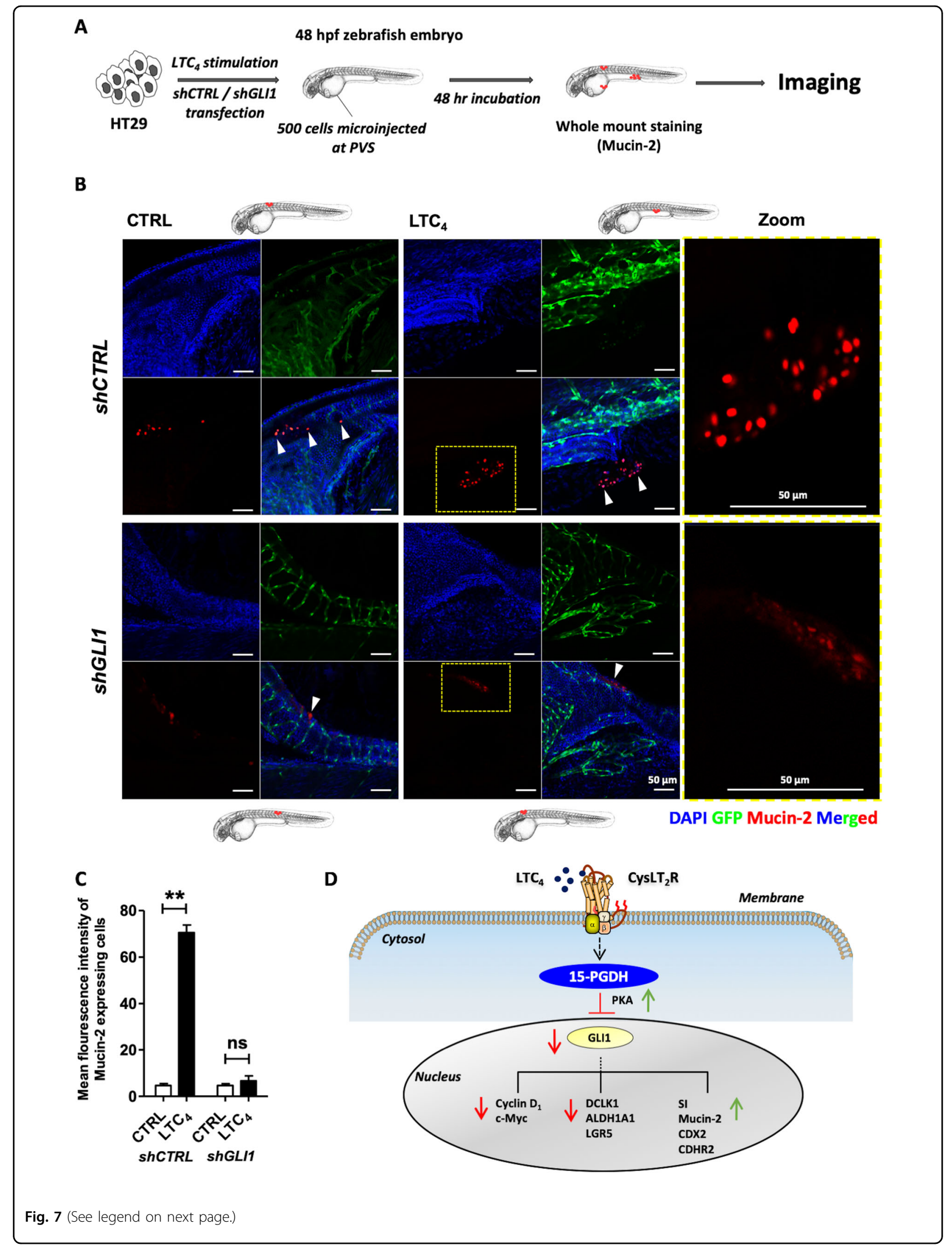


(see figure on previous page)

Fig. 7 LTC $_{4}$-induced 15-PGDH promotes differentiation in transgenic zebrafish xenografts. a Schematic diagram showing the experimental setup of HT-29 cell-based xenografts in transgenic zebrafish ( $n=20$ in each group). b Immunofluorescence analysis of whole-mount staining of xenografted transgenic zebrafish Tg(fli1:EGFP) embryos with anti-Mucin-2 antibodies after the injection of shCTRL-or shGL/1-transfected HT-29 cells with or without $\mathrm{LTC}_{4}$ stimulation into the perivitelline space (PVS) ${ }^{53}$. c Graphical representation of the mean fluorescent intensity of Mucin-2expressing HT-29 colon cancer cells transfected with ShCTRL or shGLI1 with or without $\mathrm{LTC}_{4}$ stimulation. The scale bars are as indicated in the images. d Schematic mechanistic model described in the study. Mean \pm SEM, ${ }^{* *} P<0.01$.

carcinogenesis, which was regulated through an IL-6/ STAT-3 axis that was also involved in 15-PGDH activation $^{38,39}$. These findings are in contrast with those found in the present study, where we found that 15-PGDH activation inhibited Hh signalling.

Previous studies by our group have revealed the antitumour role of $\mathrm{CysLT}_{2} \mathrm{R}$ signalling via promotion of the redifferentiation of colon cancer cells with the activation of alkaline phosphatase and Mucin-2 expression after $\mathrm{LTC}_{4}$ and IFN- $\alpha$ stimulation ${ }^{12}$. Furthermore, Bengtsson et al. emphasized the role of all-trans retinoic acid in activating $\operatorname{CysLT}_{2} \mathrm{R}$ and promoting differentiation in colon cancer cells ${ }^{40}$. An additional study by Magnusson et al. clearly demonstrated the correlation between high CysLT ${ }_{2} \mathrm{R}$ expression and both higher differentiation levels in colon cancer patients and $\mathrm{LTC}_{4}$-induced differentiation markers in colon cancer cells ${ }^{41}$. These previous studies support our current observations that $\mathrm{LTC}_{4}$-mediated CysLT $_{2} \mathrm{R}$ activation promoted a redifferentiation state, which seems to be regulated by GLI1. Recently, Mehdawi et al. reported that the activation of $\mathrm{LTC}_{4}$-induced CysLT $_{2} \mathrm{R}$ signalling led to the activation of $15-\mathrm{PGDH}$, which further promoted the differentiation of colon cancer cells in a 15-PGDH-dependent manner, as evidenced by the upregulation of intestinal differentiation markers ${ }^{20}$. The current study determined a hitherto unknown underlying regulatory mechanism of the $\mathrm{LTC}_{4}$-induced 15-PGDH-mediated promotion of redifferentiation in colon cancer cells.

It is reasonable to speculate that a decrease in proliferation and stemness would be observed in cells undergoing differentiation. Moreover, Wnt signalling is known to contribute greatly to proliferation and stemness in CRC cells. Based on this background, we hypothesised that $\mathrm{LTC}_{4}$-mediated 15-PGDH induction may also have a negative effect on Wnt target proteins. We therefore investigated the mRNA expression of the Wnt target genes AXIN2, $c M Y C$, and CYCLIND1, which are closely associated with cellular proliferation and stemness enrichment. $\mathrm{LTC}_{4}$-induced 15-PGDH downregulated Wnt target genes in a GLI1-dependent manner with concurrent activation of the intestinal tumour suppressor $C D X 2$, which positively regulates the expression of SI in colon cancer cells ${ }^{24,42}$ and is a prominent intestinal differentiation marker. In adult intestines, CDX2 controls the balance between proliferation and differentiation, and its depletion results in dedifferentiation, leading to cancer pathogenesis $^{43,44}$. In CRC, CDX2 is highly suppressed ${ }^{45}$ and is inversely correlated with disease progression and metastasis $^{46,47}$. However, previous reports suggested that activated $C D X 2$ regulates AXIN2 expression in Caco-2 cells $^{42,48}$. Moreover, it regulates the Wnt degradation complex consisting of AXIN2, APC/GSK-3 $\beta$, and CK-1, and it re-establishes a differentiation state ${ }^{49}$. The relationship between CDX2 and CYCLIND1 and the $C M Y C$ oncogene has been elucidated previously ${ }^{50,51}$. Taken together, our observations support the positive correlation between $C D X 2$ and $S I$ as well as the negative regulatory action of $C D X 2$ on the designated Wnt targets AXIN2, $c M Y C$, and CYCLIND1 in colon cancer cells.

Our in vivo model results suggest a negative correlation between GLI1 expression and $\mathrm{CysLT}_{2} \mathrm{R}$ signalling. Moreover, IHC analysis of CRC patient tissues showed a significant negative correlation between GLI1, 15-PGDH and Mucin-2 expression in CRC tissue, which is in line with the mRNA data from the public CRC database. Although we did not observe a significant correlation between GLI1 and CysLT $_{2} \mathrm{R}$ expression in our patient cohort, the public data from Sveen et al. show a weakly negative but significant correlation between the mRNA expression levels of GLI1 and CYSLTR2, which is further supported by our in vitro and in vivo findings. Furthermore, the mRNA analysis of paired normal and cancer tissues from patients suggested that GLI1 and stemness were positively correlated (Figs. 1l, 6l, n) and coupled with adverse effects on differentiation-promoting factors (Fig. 2c). The protein expression of $\mathrm{CysLT}_{2} \mathrm{R}$ and 15PGDH in matched normal vs tumour tissue showed a negative correlation with GLI1 expression (Fig. 1k). Therefore, the $\mathrm{LTC}_{4} / \mathrm{CysLT}_{2} \mathrm{R} / 15-\mathrm{PGDH}$ signallingmediated downregulation of Hh-GLI1 signalling combined with increased redifferentiation could be an alternative therapeutic treatment in addition to classical chemotherapy for colon cancer patients (Fig. 7d).

According to this concept, we speculate that inducing 15-PGDH signalling via $\mathrm{CysLT}_{2} \mathrm{R}$ activation in CRC patients might lead to new treatment opportunities ${ }^{52}$ and better clinical outcomes by downregulating GLI1 and hence guiding the cancer cells into a more redifferentiated state. 


\section{Materials and methods}

Antibodies and reagents

Please see Supplementary Materials and Methods.

\section{Animal care and handling}

Please see Supplementary Materials and Methods.

\section{Colonosphere formation and quantification}

Please see Supplementary Materials and Methods.

\section{Dox-inducible CysLT2R stable knockout cell line}

Please see Supplementary Materials and Methods.

\section{Cell lines and reagents}

Please see Supplementary Materials and Methods.

\section{Western blot analysis}

Please see Supplementary Materials and Methods.

\section{Immunofluorescence analysis}

Please see Supplementary Materials and Methods.

Immunohistochemistry

Please see Supplementary Materials and Methods.

\section{Patients}

Please see Supplementary Materials and Methods.

\section{Ethical statement}

Please see Supplementary Materials and Methods.

\section{Public database}

Please see Supplementary Materials and Methods.

\section{Quantitative real-time PCR}

Please see Supplementary Materials and Methods.

\section{Short-hairpin RNA (shRNA) and plasmid transfection} Please see Supplementary Materials and Methods.

\section{Zebrafish xenografts}

Please see Supplementary Materials and Methods.

\section{Statistical analysis}

Please see Supplementary Materials and Methods.

\section{Acknowledgements}

We would like to thank Prof. Rune Toftgård at the Karolinska Institute, Stockholm, Sweden, for the kind gift of the pEGFP-hGL $11^{51}$ construct used in this study and acknowledge Kristina Holka in the TMA Centre at Lund University, Malmö, Sweden, for her assistance with immunohistochemistry. We also would like to thank Dr. J. Evans, Amira Pharmaceuticals (San Diego, CA, USA), for kindly providing the Cys $\mathrm{T}_{2} \mathrm{R}$ antagonist AP100984 used in this study. Open access funding provided by Lund University.
Conflict of interest

The authors declare that they have no conflict of interest.

Publisher's note

Springer Nature remains neutral with regard to jurisdictional claims in published maps and institutional affiliations.

Supplementary Information accompanies this paper at (https://doi.org/ 10.1038/s41389-020-00256-0).

Received: 3 January 2020 Revised: 14 July 2020 Accepted: 29 July 2020 Published online: 19 August 2020

\section{References}

1. Siegel, R. L. et al. Colorectal cancer statistics, 2017. CA Cancer J. Clin. 67, 177-193 (2017).

2. Katoh, Y. \& Katoh, M. Hedgehog target genes: mechanisms of carcinogenesis induced by aberrant hedgehog signaling activation. Curr. Mol. Med. 9, 873-886 (2009).

3. Mishra, S., Bernal, C., Silvano, M., Anand, S. \& Ruiz, I. A. A. The protein secretion modulator TMED9 drives CNIH4/TGFalpha/GLI signaling opposing TMED3WNT-TCF to promote colon cancer metastases. Oncogene $\mathbf{3 8}$, 5817-5837 (2019).

4. Varnat, F. et al. Human colon cancer epithelial cells harbour active HEDGEHOG-GLI signalling that is essential for tumour growth, recurrence, metastasis and stem cell survival and expansion. EMBO Mol. Med 1, 338-351 (2009).

5. Madison, B. B. et al. Epithelial hedgehog signals pattern the intestinal cryptvillus axis. Development 132, 279-289 (2005).

6. Petrova, R. \& Joyner, A. L. Roles for Hedgehog signaling in adult organ homeostasis and repair. Development 141, 3445-3457 (2014).

7. Mazumdar, T. et al. Hedgehog signaling drives cellular survival in human colon carcinoma cells. Cancer Res. 71, 1092-1102 (2011).

8. Alinger, B. et al. Hedgehog signaling is involved in differentiation of normal colonic tissue rather than in tumor proliferation. Virchows Arch. 454, 369-379 (2009).

9. Chatel, G et al. Hedgehog signaling pathway is inactive in colorectal cancer cell lines. Int J. Cancer 121, 2622-2627 (2007).

10. Heise, C. E. et al. Characterization of the human cysteinyl leukotriene 2 receptor. J. Biol. Chem. 275, 30531-30536 (2000)

11. Lynch, K. R. et al. Characterization of the human cysteinyl leukotriene CysLT1 receptor. Nature 399, 789-793 (1999).

12. Magnusson, $\mathrm{C}$. et al. Low expression of CysLT1R and high expression of CysLT2R mediate good prognosis in colorectal cancer. Eur. J. Cancer $\mathbf{4 6}$ 826-835 (2010)

13. Savari, S. et al. Cysteinyl leukotriene 1 receptor influences intestinal polyp incidence in a gender-specific manner in the ApcMin/+ mouse model. Carcinogenesis 37, 491-499 (2016).

14. Wang, D. \& DuBois, R. N. Role of prostanoids in gastrointestinal cancer. J. Clin Investig 128, 2732-2742 (2018).

15. Greenhough, A. et al. The COX-2/PGE2 pathway: key roles in the hallmarks of cancer and adaptation to the tumour microenvironment. Carcinogenesis $\mathbf{3 0}$ 377-386 (2009).

16. Backlund, M. G. et al. 15-Hydroxyprostaglandin dehydrogenase is downregulated in colorectal cancer. J. Biol. Chem. 280, 3217-3223 (2005).

17. Mann, J. R. et al. Repression of prostaglandin dehydrogenase by epiderma growth factor and snail increases prostaglandin E2 and promotes cancer progression. Cancer Res. 66, 6649-6656 (2006).

18. Gustafsson, A. et al. Receptor and enzyme expression for prostanoid metabolism in colorectal cancer related to tumor tissue PGE2. Int J. Oncol. 36 469-478 (2010)

19. Mehdawi, L. M., Prasad, C. P., Ehrnstrom, R., Andersson, T. \& Sjolander, A. Noncanonical WNT5A signaling up-regulates the expression of the tumor suppressor 15-PGDH and induces differentiation of colon cancer cells. Mol. Oncol 10, 1415-1429 (2016)

20. Mehdawi, L. M. et al. A potential anti-tumor effect of leukotriene C4 through the induction of 15-hydroxyprostaglandin dehydrogenase expression in colon cancer cells. Oncotarget. 8, 35033-35047 (2017). 
21. Myung, S. J. et al. 15-Hydroxyprostaglandin dehydrogenase is an in vivo suppressor of colon tumorigenesis. Proc. Natl Acad. Sci. USA 103, 12098-12102 (2006).

22. Topi, G. et al. Association of the oestrogen receptor beta with hormone status and prognosis in a cohort of female patients with colorectal cancer. Eur. J. Cancer 83, 279-289 (2017).

23. Sveen, A. et al. Transcriptome instability in colorectal cancer identified by exon microarray analyses: Associations with splicing factor expression levels and patient survival. Genome Med 3, 32 (2011).

24. Osman, J. et al. Cysteinyl leukotriene receptor 1 facilitates tumorigenesis in a mouse model of colitis-associated colon cancer. Oncotarget 8, 34773-34786 (2017).

25. Kaesler, S., Luscher, B. \& Ruther, U. Transcriptional activity of GLI1 is negatively regulated by protein kinase A. Biol. Chem. 381, 545-551 (2000).

26. Sheng, T., Chi, S., Zhang, X. \& Xie, J. Regulation of Gli1 localization by the CAMP/protein kinase A signaling axis through a site near the nuclear localization signal. J. Biol. Chem. 281, 9-12 (2006).

27. Chi, T. Y., Chen, G. G., Ho, L. K. \& Lai, P. B. Establishment of a doxycyclineregulated cell line with inducible, doubly-stable expression of the wild-type p53 gene from p53-deleted hepatocellular carcinoma cells. Cancer Cell Int. 5, 27 (2005).

28. Ek, F. et al. Behavioral analysis of dopaminergic activation in zebrafish and rats reveals similar phenotypes. ACS Chem. Neurosci. 7, 633-646 (2016).

29. Merchant, J. L. Hedgehog signalling in gut development, physiology and cancer. J. Physiol. 590, 421-432 (2012).

30. Gerling, M. et al. Stromal Hedgehog signalling is downregulated in colon cancer and its restoration restrains tumour growth. Nat. Commun. 7, 12321 (2016).

31. Lee, J. J. et al. Control of inflammation by stromal Hedgehog pathway activation restrains colitis. Proc. Natl Acad. Sci. USA 113, E7545-E7553 (2016)

32. Nayak, A. et al. Nanoquinacrine induced apoptosis in cervical cancer stem cells through the inhibition of hedgehog-GLI1 cascade: Role of GLI-1. Sci. Rep. $\mathbf{6}$, 20600 (2016).

33. Arima, K. et al. Inhibition of 15-PGDH causes Kras-driven tumor expansion through prostaglandin E2-ALDH1 signaling in the pancreas. Oncogene $\mathbf{3 8}$ 1211-1224 (2019).

34. Hughes, D. et al. NAD+-dependent 15-hydroxyprostaglandin dehydrogenase regulates levels of bioactive lipids in non-small cell lung cancer. Cancer Prev. Res. 1, 241-249 (2008).

35. Seira, N. et al. Anti-cancer effects of MW-03, a novel indole compound, by inducing 15-hydroxyprostaglandin dehydrogenase and cellular growth inhibition in the LS174T human colon cancer cell line. Biol. Pharm. Bull. 40, 1806-1812 (2017).

36. Choi, S. H. et al. Synthetic triterpenoid induces 15-PGDH expression and suppresses inflammation-driven colon carcinogenesis. J. Clin. Investig 124, 2472-2482 (2014).

37. Fink, S. P. et al. Sulindac reversal of 15-PGDH-mediated resistance to colon tumor chemoprevention with NSAIDs. Carcinogenesis 36, 291-298 (2015).
38. Kangwan, N. et al. Sonic hedgehog inhibitors prevent colitis-associated cancer via orchestrated mechanisms of IL-6/gp130 inhibition, 15-PGDH induction, Bcl-2 abrogation, and tumorsphere inhibition. Oncotarget 7, 7667-7682 (2016).

39. Kangwan, N. et al. Concerted actions of ameliorated colitis, aberrant crypt foci inhibition and 15-hydroxyprostaglandin dehydrogenase induction by sonic hedgehog inhibitor led to prevention of colitis-associated cancer. Int J. Cancer 138, 1482-1493 (2016).

40. Bengtsson, A. M. et al. The cysteinyl leukotriene 2 receptor contributes to alltrans retinoic acid-induced differentiation of colon cancer cells. BMC Cancer 13, 336 (2013).

41. Magnusson, C., Ehrnstrom, R., Olsen, J. \& Sjolander, A. An increased expression of cysteinyl leukotriene 2 receptor in colorectal adenocarcinomas correlates with high differentiation. Cancer Res 67, 9190-9198 (2007).

42. Olsen, A. K. et al. Regulation of APC and AXIN2 expression by intestinal tumor suppressor CDX2 in colon cancer cells. Carcinogenesis 34, 1361-1369 (2013).

43. Beck, F., Chawengsaksophak, K., Waring, P., Playford, R. J. \& Furness, J. B. Reprogramming of intestinal differentiation and intercalary regeneration in Cdx2 mutant mice. Proc. Natl Acad. Sci. USA 96, 7318-7323 (1999).

44. Chawengsaksophak, K., James, R., Hammond, V. E., Kontgen, F. \& Beck, F. Homeosis and intestinal tumours in Cdx2 mutant mice. Nature 386, 84-87 (1997).

45. Bakaris, S., Cetinkaya, A., Ezberci, F. \& Ekerbicer, H. Expression of homeodomain protein CDX2 in colorectal adenoma and adenocarcinoma. Histol. Histopathol. 23, 1043-1047 (2008).

46. Thilakasiri, P. et al. Repurposing the selective estrogen receptor modulator bazedoxifene to suppress gastrointestinal cancer growth. EMBO Mol. Med. 11, e9539 (2019).

47. Boyd, M. et al. Genome-wide analysis of CDX2 binding in intestinal epithelial cells (Caco-2). J. Biol. Chem. 285, 25115-25125 (2010).

48. Sancho, R. et al. JNK signalling modulates intestinal homeostasis and tumourigenesis in mice. Embo j. 28, 1843-1854 (2009).

49. Sakuma, K., Aoki, M. \& Kannagi, R. Transcription factors c-Myc and CDX2 mediate E-selectin ligand expression in colon cancer cells undergoing EGF/ bFGF-induced epithelial-mesenchymal transition. Proc. Natl Acad. Sci. USA 109, 7776-7781 (2012).

50. Takahashi, K., Hirano, F., Matsumoto, K., Aso, K. \& Haneda, M. Homeobox gene CDX2 inhibits human pancreatic cancer cell proliferation by down-regulating cyclin D1 transcriptional activity. Pancreas 38, 49-57 (2009).

51. Lauth, M., Bergstrom, A., Shimokawa, T. \& Toftgard, R. Inhibition of GLImediated transcription and tumor cell growth by small-molecule antagonists. Proc. Natl Acad. Sci. USA 104, 8455-8460 (2007).

52. Bellamkonda, K. et al. Montelukast, a CysLT1 receptor antagonist, reduces colon cancer stemness and tumor burden in a mouse xenograft model of human colon cancer. Cancer Lett. 437, 13-24 (2018).

53. Inoue, D. \& Wittbrodt, J. One for all-a highly efficient and versatile method for fluorescent immunostaining in fish embryos. PLOS ONE 6, e19713 (2011). 